\title{
Correspondence
}

\section{B Cell Deficiency in Patients with Relapsed and Refractory Acute Myeloid Leukemia}

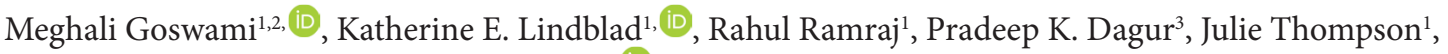 \\ J. Philip McCoy ${ }^{3}$, Christopher S. Hourigan ${ }^{1 *}$, (D) \\ ${ }^{1}$ Laboratory of Myeloid Malignancies, Hematology Branch, National Heart, Lung, and Blood Institute, National Institutes of Health, Bethesda, MD, USA \\ ${ }^{2}$ Institute for Biomedical Sciences, The George Washington University, Washington, DC, USA \\ ${ }^{3}$ Flow Cytometry Core, National Heart, Lung, and Blood Institute, National Institutes of Health, Bethesda, MD, USA
}

\author{
ARTICLE INFO \\ Article History \\ Received 23 April 2020 \\ Accepted 17 June 2020 \\ Keywords \\ AML \\ adaptive immunity \\ B lymphocyte \\ Tregs \\ PD-1 \\ immunotherapy
}

(C) 2020 International Academy for Clinical Hematology. Publishing services by Atlantis Press International B.V. This is an open access article distributed under the CC BY-NC 4.0 license (http://creativecommons.org/licenses/by-nc/4.0/).
Immune checkpoint inhibition for the treatment of acute myeloid leukemia (AML) has been investigated clinically but has not yet been shown to be more effective than the standard of care treatment [1]. Tumor-specific autologous $\mathrm{T}$ cells expressing inhibitory receptors (iRs) are an inherent foundation to this immunotherapy; thus, a detailed picture of cells infiltrating the tumor microenvironment in AML, i.e., the bone marrow (BM), is warranted [2,3]. In this study, we characterized the frequencies and immunophenotypes of adaptive immune cells in the BM of relapsed or refractory AML (R-AML) patients in tandem with age-matched healthy donors (HD) for better contextualization of immunophenotypic variation in both cohorts. We performed deep immunological profiling using multi-parameter flow cytometry of BM of 10 R-AML and 20 age-matched $\mathrm{HD}$, and considered the frequency, composition and expression of clinically relevant iRs across $\mathrm{T}$ and $\mathrm{B}$ cell subsets in HD and R-AML. Our group previously assessed the BM of this same HD cohort using single-cell RNA sequencing, mass cytometry and flow cytometry to cross-validate these technological approaches and to develop a reference data set for normal immune cell variation in healthy BM [4].

Bone marrow aspirate was collected from patients with R-AML at the National Heart, Lung, and Blood Institute (NHLBI) at the National Institutes of Health (NIH) [5,6]. Adult HD were recruited for BM aspirate collection at the $\mathrm{NIH}$; all subjects were screened on IRB-approved protocols and provided written informed consent.

"Corresponding author.Email: hourigan@nih.gov

Peer review under responsibility of the International Academy for Clinical Hematology
Purification and storage of BM mononuclear cells (BMMCs), multi-parameter flow cytometry, and data acquisition were all performed as previously described [4]. For intracellular FOXP3 staining, BMMCs were fixed and permeabilized using components of the FOXP3 Transcription Staining Buffer Set (eBioscience, Waltham, MA, USA) according to the manufacturer's protocol, stained for 30 min with FOXP3 antibody in the dark at room temperature, and washed prior to acquisition. In one R-AML patient, we were unable to distinguish between $\mathrm{T}$ cells and leukemic blasts due to apparent expression of T cell markers by the AML; we excluded this patient from subsequent T cell analyses. Two-tailed Mann-Whitney $U$-tests were used to compare variables in the two sample groups. Data analysis and visualization were performed using FlowJo 10.1 (BD, Ashland, OR, USA) and Prism8 (GraphPad, San Diego, CA, USA).

The average age of the ten R-AML patients was 53 years (range 23-68), with four females and six males. All patients had at least one cycle of chemotherapy; none had received previous immune checkpoint therapy. Demographic details on the HD cohort can be found in Oetjen et al. [4]. We had previously identified abnormal B cell phenotypic distributions and function in AML patients in remission [7]. In the current study, $\mathrm{HD}$ have an abundant population of CD19+ and CD20+ B cells in the BM, whereas this population was minimal in this group of R-AML patients with active disease (Figure 1A). To ensure this difference was not due to leukemic blast content in R-AML patients, we adjusted the number of live cells used as a denominator for quantification by the clinically reported blast percentage, and calculated B cell frequency as a percentage of live non-blast cells in R-AML. This quantification showed a dramatic and highly significant depletion of CD19+ 
A
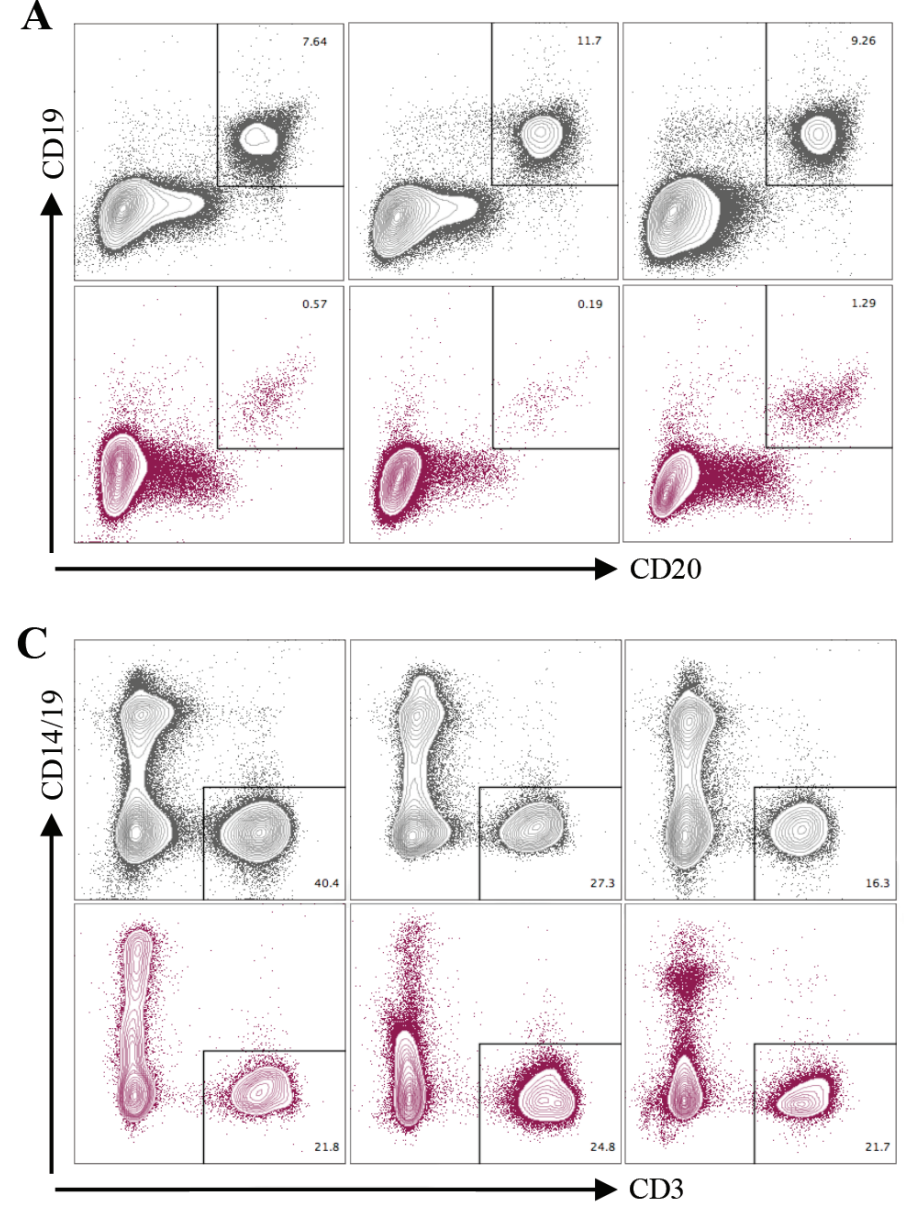

\section{D}

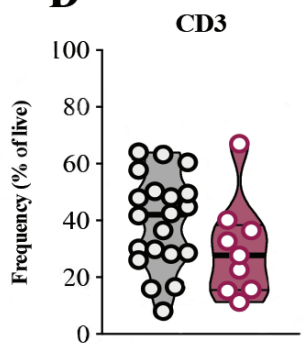

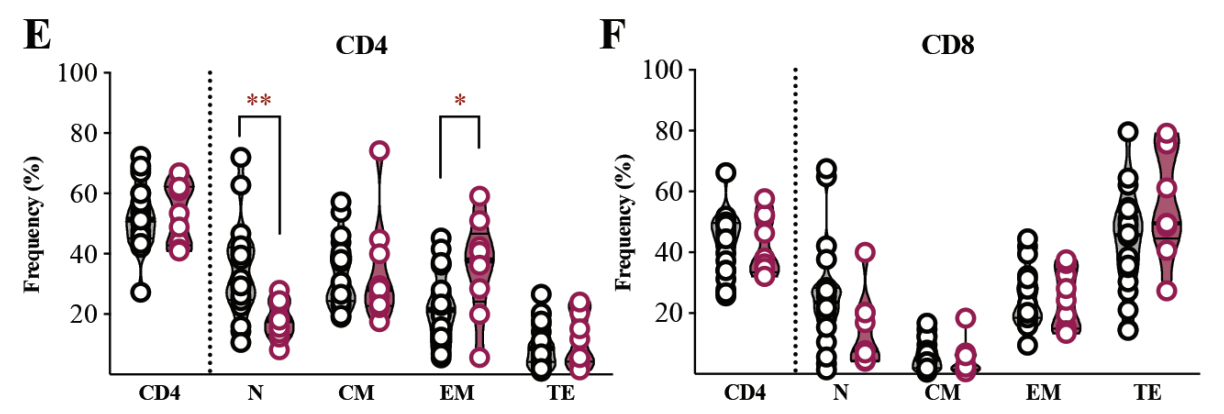
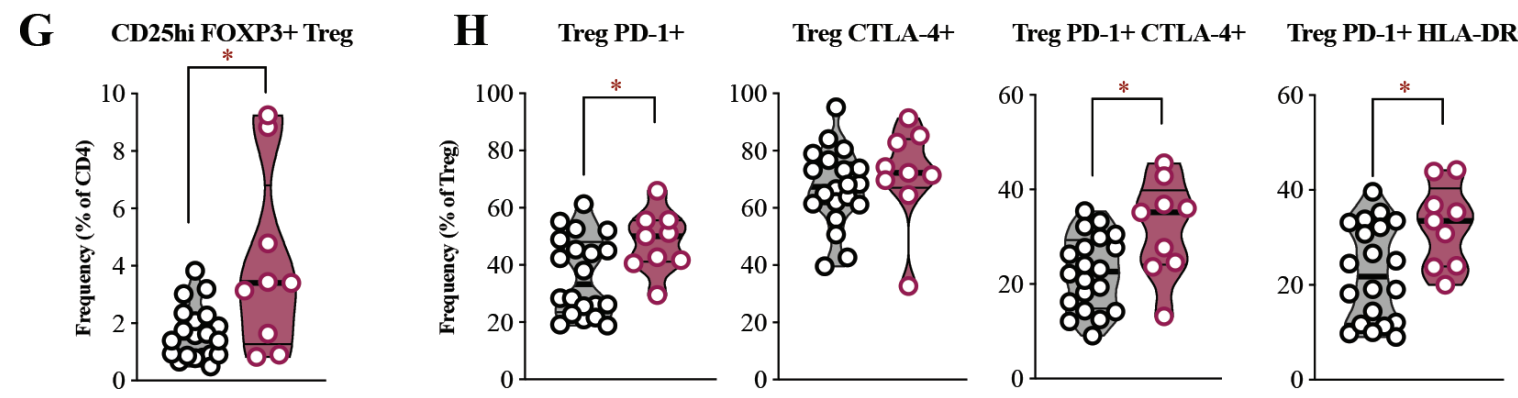

Figure 1 B cell loss but comparable T cell frequencies in R-AML versus HD. (A) Representative multi-parameter flow cytometry (MFC) plots of CD19+ and CD20+ B cells. (B) Frequencies of CD19+ B cells ( $n=10$ R-AML, $n=20$ HD). (C) Representative MFC plots of CD3+ T cells. $Y$-axis indicates CD14/ CD19 exclusion channel. (D) Frequencies of CD3+ T cells $(n=9 \mathrm{R}-\mathrm{AML}, n=20 \mathrm{HD})$. (E and F) Frequencies of CD4+ and CD8+ T cells as a percentage of $\mathrm{CD} 3+$ and naïve $(\mathrm{N})$, central memory (CM), effector memory (EM), and terminal effector (TE) as a percentage of either CD4+ or CD8+. (G) Frequency of CD25hi FOXP3+ Tregs. (H) Frequencies of Tregs expressing iRs and activation markers. For MFC plots, representative HD data in gray and R-AML in red. For boxplot panels, HD data in black and gray and R-AML in red. ${ }^{*} p<0.05 ;{ }^{* *} p<0.01 ;{ }^{* * *} p<0.001$ by Mann-Whitney tests. 
$\mathrm{B}$ cells in AML patients (mean frequency \pm standard deviation; HD: $12.4 \pm 8.3$; AML: $2.9 \pm 3.2, p<0.001$ ) (Figure 1B). The absolute numbers of CD19+ events in AML were too low to confidently quantify transitional, naive, or memory B cell subpopulations and immune checkpoints expressed by these subsets.

The reduced $\mathrm{B}$ cell frequencies in $\mathrm{BM}$ were in stark contrast to $\mathrm{CD} 3+\mathrm{T}$ cells. The average and range of $\mathrm{T}$ cell frequencies were not different between the R-AML and HD cohorts after a similar adjustment for leukemic blast burden in R-AML (HD: $39.5 \pm 16.3$; AML: $29.9 \pm 17.2$ ) (Figure $1 \mathrm{C}$ and $1 \mathrm{D}$ ). The percentages of CD4+ and CD8+ T cells as well as CD4:CD8 ratio in R-AML were well within the ranges seen in HD. We observed depressed frequencies of naïve CD4+ T cells in R-AML (HD: $39.9 \pm 14.8$; AML: $22.6 \pm 11.0$, $p<0.01$ ) and increased CD4+ EM cells (HD: $20.1 \pm 11.0$; AML: 33.6 $\pm 17.0, p<0.05$ ), although the frequencies of these populations in $\mathrm{R}$-AML largely remained within the range seen in HD (Figure 1E). The frequencies of CD8+ naïve, CM, EM, and TE in R-AML were indistinguishable from those in HD (Figure 1F). One notable difference seen, consistent with prior reports, was a higher frequency of Tregs in R-AML (HD: $1.8 \pm 1.1$; AML: $4.2 \pm 3.0, p<0.05$ ) [8-12]. Three R-AML patients in our cohort had Treg frequencies above the upper limit of that on HD (Figure $1 G$ ). Furthermore, iR analysis indicated a higher average PD-1+ expression, as well as higher co-expression of PD-1 with CTLA-4 and HLA-DR in Tregs (Figure $1 \mathrm{H}$ ). These data confirm that in some AML patients activated and, likely, immunosuppressive Tregs may be increased [13].

To examine patterns of $\mathrm{iR}$ co-expression on CD4+ and CD8+ T cells, we used Boolean gating of four iRs (PD-1, TIGIT, CTLA-4, AND TIM-3); the top seven most frequent iR combinations in CD4+ and CD8+ T cells are listed in Table 1 . We found that many

Table 1 Abundant combinatorial iR patterns in CD8+ and CD4+ $\mathrm{T}$ cells

\begin{tabular}{lccccc}
\hline & \multicolumn{2}{c}{ R-AML } & & \multicolumn{2}{c}{ HD } \\
\cline { 2 - 3 } \cline { 5 - 6 } CD8+ T cell & $\begin{array}{c}\text { Mean } \pm \\
\text { std. dev. }\end{array}$ & Range & & $\begin{array}{c}\text { Mean } \pm \\
\text { std. dev. }\end{array}$ & Range \\
\hline iR negative & $30.1 \pm 16.8$ & $4.9-60.0$ & & $27.6 \pm 12.2$ & $9.7-48.9$ \\
TIGIT+ & $15.5 \pm 9.2$ & $5.1-34.5$ & & $11.9 \pm 7.1$ & $1.0-30.3$ \\
PD-1+ TIGIT+ & $11.7 \pm 6.3$ & $3.8-22.2$ & & $11.3 \pm 7.2$ & $1.5-26.9$ \\
CTLA-4+ & $7.8 \pm 8.0$ & $0.6-27.0$ & & $14.7 \pm 14.0$ & $1.3-52.7$ \\
PD-1+ CTLA-4+ TIGIT+ & $7.8 \pm 6.2$ & $0.4-20.3$ & & $7.9 \pm 5.6$ & $0.4-23.8$ \\
CTLA-4+ TIGIT+ & $7.2 \pm 6.1$ & $1.0-20.7$ & & $7.5 \pm 6.4$ & $1.7-22.6$ \\
PD-1+ & $6.2 \pm 3.0$ & $1.7-11.6$ & & $6.7 \pm 3.8$ & $0.8-16.2$ \\
\hline & \multicolumn{2}{c}{ R-AML } & & & HD \\
\cline { 2 - 3 } \cline { 5 - 6 } CD4+ T cell & Mean \pm & & & Mean \pm & \\
& std. dev. & Range & & std. dev. & Range \\
iR negative & $48.6 \pm 8.0$ & $39.9-61.6$ & & $58.9 \pm 9.4$ & $40.1-76.5$ \\
PD-1+ & $14.6 \pm 7.7$ & $3.6-28.5$ & & $10.7 \pm 6.5$ & $2.5-31.5$ \\
TIGIT+ & $9.9 \pm 6.3$ & $4.0-23.4$ & & $6.2 \pm 3.1$ & $2.5-14.5$ \\
PD-1+ TIGIT+ & $7.3 \pm 1.7$ & $4.1-10.0$ & & $5.5 \pm 2.9$ & $1.2-11.8$ \\
CTLA-4+ & $4.2 \pm 2.4$ & $1.1-8.7$ & & $4.0 \pm 2.3$ & $1.5-11.7$ \\
TIM-3+ & $4.1 \pm 1.6$ & $2.2-6.9$ & & $6.4 \pm 3.5$ & $1.4-16.5$ \\
PD-1+ CTLA4+ & $3.3 \pm 2.3$ & $0.4-7.2$ & & $2.4 \pm 1.2$ & $0.6-5.5$ \\
\hline
\end{tabular}

All values represent percentages. Std. dev, indicates standard deviation; iR, inhibitory receptor.
CD4+ $\mathrm{T}$ cells in HD and R-AML did not express any of these iRs. Across HD, as few as $9.7 \%$ and as many as $48.9 \%$ of CD8+ $\mathrm{T}$ cells did not express any of the tested iRs, with a greater range in R-AML (4.9-60.0\%). Within the CD4+ compartment of both HD and R-AML, PD-1 was the most commonly singularly expressed $\mathrm{iR}$, followed by TIGIT. In the CD8+ compartment, TIGIT was the most abundantly singularly expressed iR. PD-1 and TIGIT were the most commonly co-expressed iRs in both CD4+ and CD8+ $\mathrm{T}$ cells, and frequencies of cells co-expressing these markers were highly similar in R-AML and HD. Of note, we found that $7.9 \pm 5.6 \%$ and $7.8 \pm 6.2 \%$ of CD $8+\mathrm{T}$ cells from HD and R-AML, respectively, co-expressed PD-1, TIGIT, and CTLA-4 (Table 1). These cells were predominantly EM and TE phenotypes in patients and HD. Other combinations of iRs outside those listed on Table 1 were rare and accounted for $<5 \%$ of CD $4+$ or CD8+ $\mathrm{T}$ cells. These data highlight common iR co-expression patterns as well as the overall degree of similarity in terms of iR expression in R-AML and our HD cohort.

In summary, we have shown that R-AML patients have marked reductions of $\mathrm{CD} 19+\mathrm{B}$ cells in their $\mathrm{BM}$. This was striking, given the preservation of the $\mathrm{T}$ cell compartment in R-AML. A subset of R-AML patients had elevated frequencies of Tregs, but the nonTreg T cell subsets in R-AML were similar to those seen in a cohort of age- and gender-matched HD. Our data on T cell subset frequencies in R-AML are consistent with those previously published, but place these findings in the context of the wide variation that occurs in healthy individuals during aging $[14,15]$. These data also expand our previous analysis of the immune infiltrate in the BM of $\mathrm{HD}$, by adding iR expression information on $\mathrm{T}$ cell subsets [4]. Together, our results reveal a preserved pool of antigen-experienced $\mathrm{T}$ cells expressing PD-1 and other iRs within the BM of R-AML. The observed depletion of B cells in these patients' BM may have consequences for immunotherapies intended to augment existing anti-tumour immunity [16-19].

\section{CONFLICTS OF INTEREST}

CSH receives research funding from Merck, Sharpe, \& Dohme and SELLAS Life Sciences Group AG. The other authors declare no conflicts of interest.

\section{AUTHORS' CONTRIBUTION}

MG designed and performed experiments, analyzed data, wrote the manuscript, and prepared the figures. KEL performed experiments and analyzed data. RR analyzed data. PKD and JPM designed and supervised the research. JT cared for patients and coordinated donor recruitment. CSH cared for patients, designed and supervised the research, and edited the manuscript. All authors read, reviewed, and approved the final manuscript.

\section{FUNDING}

This research was supported by the Intramural Research Program of NHLBI at the NIH. 


\section{ACKNOWLEDGMENTS}

We are grateful to Catherine Lai, Sheenu Sheela, Christin DeStefano, Janet Valdez, and the NHLBI research nurses for caring for patients and bone marrow aspirate procurement and to Karolyn Oetjen for technical input. This work is part of Meghali Goswami's dissertation in partial fulfillment of the requirements for the $\mathrm{PhD}$ degree in the Molecular Medicine Program of the Institute for Biomedical Sciences at The George Washington University.

\section{REFERENCES}

[1] Daver N, Garcia-Manero G, Basu S, Boddu PC, Alfayez M, Cortes JE, et al. Efficacy, safety, and biomarkers of response to azacitidine and nivolumab in relapsed/refractory acute myeloid leukemia: a nonrandomized, open-label, phase II study. Cancer Discov 2019;9;370-83.

[2] Percival ME, Lai C, Estey E, Hourigan CS. Bone marrow evaluation for diagnosis and monitoring of acute myeloid leukemia. Blood Rev 2017;31;185-92.

[3] Tumeh PC, Harview CL, Yearley JH, Shintaku IP, Taylor EJ, Robert L, et al. PD-1 blockade induces responses by inhibiting adaptive immune resistance. Nature 2014;515;568-71.

[4] Oetjen KA, Lindblad KE, Goswami M, Gui G, Dagur PK, Lai C, et al. Human bone marrow assessment by single-cell RNA sequencing, mass cytometry, and flow cytometry. JCI Insight 2018;3;e124928.

[5] Wong HY, Sung AD, Lindblad KE, Sheela S, Roloff GW, Rizzieri D, et al. Molecular measurable residual disease testing of blood during AML cytotoxic therapy for early prediction of clinical response. Front Oncol 2019;8;669.

[6] Popescu B, Sheela S, Thompson J, Grasmeder S, Intrater T, DeStefano CB, et al. Timed sequential salvage chemotherapy for relapsed or refractory acute myeloid leukemia. Clin Hematol Int 2020;2;27-31.

[7] Goswami M, Prince G, Biancotto A, Moir S, Kardava L, Santich $\mathrm{BH}$, et al. Impaired B cell immunity in acute myeloid leukemia patients after chemotherapy. J Transl Med 2017;15;155.

[8] Wang X, Zheng J, Liu J, Yao J, He Y, Li X, et al. Increased population of $\mathrm{CD} 4^{+} \mathrm{CD} 25^{\text {high }}$ regulatory $\mathrm{T}$ cells with their higher apoptotic and proliferating status in peripheral blood of acute myeloid leukemia patients. Eur J Haematol 2005;75;468-76.
[9] Kanakry CG, Hess AD, Gocke CD, Thoburn C, Kos F, Meyer C, et al. Early lymphocyte recovery after intensive timed sequential chemotherapy for acute myelogenous leukemia: peripheral oligoclonal expansion of regulatory $\mathrm{T}$ cells. Blood 2011;117;608-17.

[10] Szczepanski MJ, Szajnik M, Czystowska M, Mandapathil M, Strauss L, Welsh A, et al. Increased frequency and suppression by regulatory $\mathrm{T}$ cells in patients with acute myelogenous leukemia. Clin Cancer Res 2009;15;3325-32.

[11] Shenghui Z, Yixiang H, Jianbo W, Kang Y, Laixi B, Yan Z, et al. Elevated frequencies of $\mathrm{CD} 4^{+} \mathrm{CD} 25^{+} \mathrm{CD} 127^{\text {lo }}$ regulatory $\mathrm{T}$ cells is associated to poor prognosis in patients with acute myeloid leukemia. Int J Cancer 2011;129;1373-81.

[12] Lichtenegger FS, Lorenz R, Gellhaus K, Hiddemann W, Beck B, Subklewe M. Impaired NK cells and increased T regulatory cell numbers during cytotoxic maintenance therapy in AML. Leuk Res 2014;38;964-9.

[13] Togashi Y, Shitara K, Nishikawa H. Regulatory T cells in cancer immunosuppression - implications for anticancer therapy. Nat Rev Clin Oncol 2019;16;356-71.

[14] Williams P, Basu S, Garcia-Manero G, Hourigan CS, Oetjen KA, Cortes JE, et al. The distribution of T-cell subsets and the expression of immune checkpoint receptors and ligands in patients with newly diagnosed and relapsed acute myeloid leukemia. Cancer 2019;125;1470-81.

[15] Schnorfeil FM, Lichtenegger FS, Emmerig K, Schlueter M, Neitz JS, Draenert R, et al. T cells are functionally not impaired in AML: increased PD-1 expression is only seen at time of relapse and correlates with a shift towards the memory $\mathrm{T}$ cell compartment. J Hematol Oncol 2015;8;93.

[16] Griss J, Bauer W, Wagner C, Simon M, Chen M, GrabmeierPfistershammer K, et al. B cells sustain inflammation and predict response to immune checkpoint blockade in human melanoma. Nat Commun 2019;10;4186.

[17] Cabrita R, Lauss M, Sanna A, Donia M, Skaarup Larsen M, Mitra S, et al. Tertiary lymphoid structures improve immunotherapy and survival in melanoma. Nature 2020;577;561-5.

[18] Helmink BA, Reddy SM, Gao J, Zhang S, Basar R, Thakur R, et al. B cells and tertiary lymphoid structures promote immunotherapy response. Nature 2020;577;549-55.

[19] Petitprez F, de Reyniès A, Keung EZ, Chen TWW, Sun CM, Calderaro J, et al. B cells are associated with survival and immunotherapy response in sarcoma. Nature 2020;577;556-60. 